\title{
Effects of loneliness and social isolation on sleep health
}

\author{
Yoko Komada ${ }^{1}$
}

Published online: 25 February 2022

(c) The Author(s), under exclusive licence to Japanese Society of Sleep Research 2022

Loneliness and social isolation markedly increase mortality risk, and are linked to numerous mental and physical comorbidities. Intrapersonal distress and self-reported loneliness are linked to worse sleep quality, specifically lower sleep efficiency, while active socializing is associated with better sleep quality [1]. Under COVID-19 mandated social restrictions, decrements of outdoor-light exposure and sleep duration have led to dose-dependent deterioration in quality of life [2].

The terms loneliness and social isolation are distinguished because they are different. However, these terms are often used interchangeably in academic literature. Some people who experience loneliness can also be socially isolated, whereas other people who are socially isolated do not always experience being lonely. However, racial and sexual minorities have significantly smaller social networks and lower levels of interaction with other community members than non-minorities. In addition, certain life events such as moving and divorce increase the risk of feeling lonely more often [3].

The marital relationship has a significant impact on sleep problems. For example, those who lost their spouses/partners in later life were reported to have poorer sleep quality than those who had not [4]. A cross-sectional survey of day workers (578 men and 208 women, 18-65 years old) published in Sleep and Biological Rhythms [5] has shown that being unmarried is significantly related to poor sleep phase, quality, and quantity using the 3-Dimensional Sleep Scale (3DSS). In unmarried participants, odds ratios (OR) were highest for poor sleep quantity in men (OR: 2.15) and poor sleep phase in women (OR: 2.73). Low scores in all three-dimensional aspects, i.e., sleep quality, sleep quantity, and sleep phase, showed the highest odds ratios for unmarried men (OR: 2.74) and women (OR: 6.13). In addition, sleep problems, insomnia, and overall sleep disorders are

Yoko Komada

komay@my-pharm.ac.jp

1 Liberal Arts, Meiji Pharmaceutical University, Tokyo, Japan associated with suicidal ideation independently of depression and anxiety [6]. These findings suggest the importance of creating policies to promote adequate sleep and good mental health in unmarried people.

Loss of social contact is exceedingly damaging to our humanity, well-being, and health. Indeed, current research shows that loneliness damages our physical health, including raising the risk of sleep problems. Of course, loneliness is not new, but we should recognize and address loneliness as one of the most pressing public health issues.

\section{References}

1. Ben Simon E, Walker MP. Sleep loss causes social withdrawal and loneliness. Nat Commun. 2018;9:3146.

2. Korman M, Tkachev V, Reis C, Komada Y, Kitamura S, Gubin D, Kumar V, Roenneberg T. Outdoor daylight exposure and longer sleep promote wellbeing under COVID-19 mandated restrictions. J Sleep Res. 2021;21: e13471.

3. HM Government. A connected society. A strategy for tackling loneliness-layint the foundations for change. 2018; Available at www.gov.uk/government/collections/governments-work-on-tackl ingloneliness

4. Stafford M, Bendayan R, Tymoszuk U, Kuh D. Social support from the closest person and sleep quality in later life: evidence from a British birth cohort study. J Psychosom Res. 2017;98:1-9.

5. Matsumoto Y, Uchimura N, Ishitake T. The relationship between marital status and multifactorial sleep in Japanese day workers. Sleep Biol Rhythms. 2021;27. Online ahead of print.

6. Yoshimasu K, Sugahara H, Akamine M, Kondo T, Fujisawa K, Tokunaga S, Kiyohara C, Miyashita K, Kubo C. Sleep disorders and suicidal ideation in Japanese patients visiting a psychosomatic clinic in a university hospital. Sleep Biol Rhythms. 2006;4:137-43.

Publisher's Note Springer Nature remains neutral with regard to jurisdictional claims in published maps and institutional affiliations. 